\title{
From Mechanistic to Data-driven Models for Surgical Planning, Guidance and Simulation
}

\author{
Zeike A. Taylor \\ Fraunhofer Institut IIS Erlangen, Germany \\ z.a.taylor@sheffield.ac.uk
}

Biomechanical and biophysical models are key tools in many applications of surgical planning and optimisation, surgical guidance, and interactive simulation for training and rehearsal. The most robust and accurate models usually are those based on the relevant equations of continuum mechanics (solid, fluid, thermal, etc.), and which are generally solved with numerical methods such as FEM. Given high quality patient-specific inputs, these can enable accurate prediction of, e.g., deformations of soft tissues, flow patterns in blood vessels, energy delivery profiles around ablation devices, etc. Two main difficulties arise, however: 1) computation times can be prohibitive, especially from the point of view of clinical deployment; and 2) the requisite "high quality patient-specific inputsmay simply not be available. To address these issues, our group and collaborators are beginning to explore how data-driven approaches can be used as surrogates for full mechanistic models, both to achieve faster computation, and to either mitigate the effects of parameter uncertainty or at least better characterise its effect. We believe that by exploiting the huge advances in machine learning and related areas experienced in recent years, entirely new classes of flexible, fast, and reliable simulation techniques can be achieved. Results achieved so far to this end will be described. 